\title{
Tests de fertilité chez l'animal domestique par fécondation in vitro
}

\author{
B Marquant-Le Guienne, P Humblot
}

UNCEIA, laboratoire de fécondation in vitro, 13, rue Jouët, BP 65, 94703 Maisons-Alfort, France

(Reçu le 15 juillet 1991; accepté le 15 septembre 1991)

\begin{abstract}
Résumé - Une des applications principales de la fécondation in vitro (FIV) chez les bovins est l'évaluation du pouvoir fécondant in vitro, en complément des examens classiques du sperme. Cela peut se rapporter aux jeunes reproducteurs mâles entrant dans les centres d'insémination artificielle (IA) afin de ne pas les mettre à l'épreuve sur leur aptitude génétique zootechnique si leurs caractéristiques fécondantes sont mauvaises. II peut s'agir aussi de taureaux très améliorateurs dont on cherche à optimiser le nombre de spermatozoïdes par dose d'l A pour permettre une diffusion maximale de leurs gènes. Chez le taureau, des coefficients de corrélation élevés ont été notés entre caractéristiques de FIV et performances de terrain estimées par le taux de non retour en œstrus 60-90 j après I'IA (TNR). Dans une étude portant sur 6 taureaux Normands, un effet très significatif du mâle a été trouvé sur le TNR et sur le taux de fécondation in vitro (FIV). Dans cette série,la relation entre taux de FIV et TNR est apparue forte $(r=0.83 ; P<0.01)$. Dans une deuxième série comprenant 17 taureaux principalement de race Holstein, la relation entre les paramètres in vitro et les TNR a été tout particulièrement marquée lorsque étaient pris en compte à la fois le taux de FIV et celui de développement embryonnaire jusqu'au stade blastocyste $(r=0,73 ; P<0,05)$. Les ovaires de 3 vaches réformées ont été récupérés à l'abattoir. Vingt huit ovocytes ont été collectés à partir de ceux-ci, maturés puis fécondés in vitro (4,7 ovocytes par ovaire). Après développement embryonnaire en culture, 13 morulas ou blastocystes transférables ont été obtenus $(4,3$ embryons par vache donneuse). Ces données sont compatibles avec la possibilité d'envisager une sélection précoce des jeunes mâles sur le test de FIV en complément de ceux classiques de jugement de l'aptitude des individus à la fonction sexuelle. Avant leur généralisation, il est cependant nécessaire de tenter d'en simplifier les procédures.
\end{abstract}

fécondation in vitro / bovin / fertilité / insémination artificielle / ovocyte

Summary - Practical uses of in vitro fertilization in the bovine. Today fertilization in vitro IVF has been applied to the evaluation of the reproductive ability of young males before being included in progeny testing programs and to produce embryos from females with a high genetic potential but culled because of age or major injuries of the genital tract preventing embryo collection. A strong relationship was found between the IVF results obtained with the semen of bulls and their field fertilization rates estimated by 60-90 d post antificial insemination (AI) non retum rate (NR). In a study involving 17 bulls a highly significant effect of the bull was found on NR and on all IVF parameters (particularly on the fertilization rate and early embryonic development). The highest relationship between in vitro parameters and field NR was found when the fertilization rate was taken into account together with embryonic development until the blastocyst stage $(\mathrm{r}=0.73 ; \mathrm{P}<0.01)$. Ovaries were recovered at the slaughterhouse from 3 culled cows providing 28 oocytes, matured in vitro and submit- 
ted to IVF (4.7 oocytes per ovary). After in vitro fertilization and embryonic development, a mean of 4.3 transferable embryos was obtained per donor cow (13 morulae or blastocysts). These results show that IVF can be used as a complementary test with classical sperm investigations to select young bulls with a good reproductive potential, and to produce embryos before culling females a with a high genetic potential.

in vitro fertilization / bovine / fertility / Al / oocyte

\section{INTRODUCTION}

Un grand nombre de tests in vitro hétérologues ont été initialement utilisés pour tenter d'estimer la fertilité des taureaux. Le développement des tests homologues reposant sur des tentatives de fécondation in vitro (FIV) avec des spermatozoïdes et des ovocytes de même espèce est beaucoup plus récent. Après avoir rappelé brièvement les caractéristiques des tests classiques d'appréciation de la fertilité reposant sur des critères issus de l'analyse du sperme, nous évoquerons les résultats obtenus à partir de tests in vitro de fécondation hétérologue. Puis dans une dernière partie, nous présenterons les résultats actuels que nous avons obtenus avec les tests de FIV homologues.

\section{TESTS MORPHOLOGIQUES ET DE MOTILITÉ DES SPERMATOZOIDES}

L'évaluation microscopique de la morphologie des spermatozoïdes a permis de montrer des taux de corrélation très variables avec les taux de fertilité estimés après insémination artificielle (IA) en ferme. Elle a en effet l'inconvénient d'être à la fois subjective et influencée largement par la technique utilisée (Linford et al, 1976, Goffaux, 1968; Saacke et Marshall, 1968).

L'estimation au microscope du pourcentage de spermatozoïdes ayant une motilité progressive semble avoir une faible répétabilité intra et inter laboratoire et dépen- dante de l'échantillon de sperme. Ceci explique les faibles coefficients de corrélation entre de tels paramètres et la fertilité des taureaux (Linford et al, 1976 ; Saacke et White, 1972). Cependant, l'estimation de la motilité à l'aide d'appareils plus sophisitiqués permet d'améliorer la répétabilité de la mesure. Elle n'entraîne pas cependant d'amélioration significative des coefficients de corrélation avec la fertilité exprimée en ferme (Amann et Hammerstedt, 1980).

D'autres tests ont été préconisés, parmi lesquels la pénétration des spermatozoïdes dans des substituts de mucus cervical tel que le gel de polyacrylamide (Lorton et al, 1981) ou l'acide hyaluronique (Galli et al, 1991). Ils n'ont pas permis cependant de mettre en évidence de relation significative entre ces résultats et la fertilité in vivo.

Cette absence de relation globale entre paramètres du sperme et fertilité a également été rapportée pour divers dosages d'enzymes acrosomiques libérées par les spermatozoïdes tel que celui du GOT (Glutamine oxaloacétate transaminase) par Pace et Graham (1970). Ces derniers tests (tableau I) permettent cependant de caractériser certains individus parmi les plus mauvais reproducteurs (Linford et al, 1976; Humblot et al, 1986).

\section{TESTS DE FÉCONDATION IN VITRO HÉTÉROLOGUES}

La pénétration in vitro d'ovocytes dépellucidés de hamster a été largement utilisée 
Tableau I. Caractéristiques du sperme après décongélation chez deux taureaux aux taux de non retour (TNR) très différents (selon Humblot et al, 1986).

\begin{tabular}{llcr} 
Taureau & $\begin{array}{l}\text { TNR } \\
(\%)\end{array}$ & $\begin{array}{c}\text { Spz mobiles } \\
(\%)\end{array}$ & $\begin{array}{r}\text { Activité GOT } \\
(*)\end{array}$ \\
\hline A & 68,1 & 56 & 21,3 \\
B & 50,2 & 30 & 8,4 \\
\hline
\end{tabular}

* Test de l'activité glutamine oxaloacetate transaminase permettant d'estimer l'intégrité de la membrane de la tête des spermatozoïdes après décongélation.

pour tester la fertilité du sperme humain sans qu'une corrélation claire n'ait pu être mise en évidence (Junca et al, 1982; Margalioth et al, 1983). Ce test a été également utilisé avec le sperme de taureau congelé et capacité dans du milieu de Brackett (Brackett et al, 1982). Après utilisation du sperme de deux taureaux de fertilité connue, il a été mis en évidence une relation inverse entre la fécondation in vitro hétérologue et le taux de Non Retour à 6090 jours (tableau II). II semblerait que le sperme du taureau le moins fécondant après IA pénètre le plus facilement les ovocytes de hamster et que la fréquence de formation du pronucléus mâle soit plus élevée pour ce taureau.

Le traitement des spermatozoïdes par des liposomes de dilaurylphosphatidylcholine (PC12) a été également utilisé par Graham et Foote (1987). II semble que ce test permette de discriminer le taureau de faible fertilité (TNR : 48,2\%) du groupe de 2 taureaux de fertilité élevée (TNR : $75,3 \%$ ). En effet avec le sperme de faible fertilité, le taux de pénétration maximal des ovocytes de hamster est atteint après traitement avec des concentrations de liposomes plus faibles (fig 1). Cependant, pour
Tableau II. Résultats de fécondation in vitro par un test hétérologue (ovocytes de hamster; spermatozoïdes de taureau) avec du sperme congelé (selon Brackett et al, 1982).

\begin{tabular}{llll} 
Taureau & $\begin{array}{l}\text { TNR (\%) } \\
(60-90 j)\end{array}$ & $\begin{array}{l}\text { Interaction } \\
(\%)\end{array}$ & $\begin{array}{l}\text { Pronucleus } \\
\text { mâle (\%) }\end{array}$ \\
\hline & & & \\
A & 69,3 & 53,8 & 38,5 \\
B & 66,3 & 84,9 & 56,6 \\
\hline
\end{tabular}

ces techniques de FIV hétérologues, le faible nombre d'animaux et l'absence d'expériences ultérieures utilisant la même méthodologie laissent planer des doutes sur la fiabilité des résultats observés.

\section{TESTS DE FÉCONDATION IN VITRO HOMOLOGUES}

La fécondation in vitro, chez les bovins, s'est développée depuis 1982. Des spermatozoides éjaculés frais ont d'abord été utilisés, puis la technique a été étendue au sperme congelé. Cependant le petit nombre d'ovocytes disponibles rendait impossible toute application de cette technique à des fins zootechniques. Les techniques de maturation des ovocytes in vitro ont ensuite été améliorées, notamment par l'addition de cellules de granulosa dans le milieu de culture. La présence de ces cellules permet aux ovocytes d'entamer un développement embryonnaire apparemment normal après fécondation in vitro (Lu et al, 1987; Fassi-Firhi et al, 1991). Il devenait dès lors possible de prélever les ovocytes dans les petits follicules d'ovaires recueillis aux abattoirs. La disponibilité d'un 


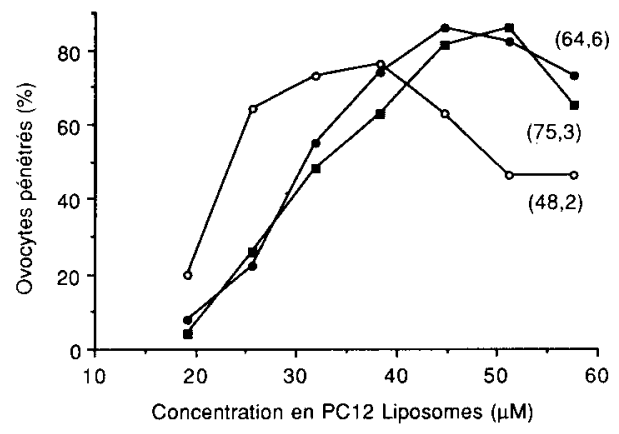

Fig 1. Pourcentage d'ovocytes de hamster pénétrés par la semence décongelée de taureaux à très faible, faible ou fort taux de non retour (48,2; 64,6 et $75,3 \%$, respectivement), après traitement avec différentes doses de PC12 liposomes (d'après Graham et Foote, 1987).

plus grand nombre d'ovocytes a donc permis d'envisager la réalisation de tests de FIV homologues pour évaluer la fertilité des taureaux.

Les premières tentatives ont été réalisées par Ohgoda et al, 1988 (tableau III). Ces auteurs ont utilisé du sperme congelé capacité dans du milieu de Brackett en présence de caféine. Ils ont observé une variation du taux de pénétration de 0 à $83 \%$ mais sans relation avec la variation du TNR. Toutefois, quelques réserves peuvent être émises vis-à-vis de ces premiers résultats. Le nombre d'IA utilisées pour apprécier la fertilité des taureaux sur le terrain est trop faible pour estimer correctement la valeur réelle de l'individu. En outre, certaines indications font penser que le milieu de fécondation utilisé n'est pas optimal pour réaliser de tels tests. II a été montré que les glycosaminoglycans (GAG'S) contenus dans le liquide folliculaire et les voies génitales femelles sont capables d'induire la réaction acrosomique du spermatozoïde de taureau (Lenz et al, 1982; Handrow et al, 1986; Parrish et al,
1985; 1988). Parmi ceux-ci, l'héparine est un des plus actifs (Handrow et al, 1982). Elle agit de manière dose-dépendante sur la réaction acrosomique (fig 2) (Lee et al, 1985). Cependant les modalités de cette réponse (\% de réaction acrosomique) sont variables. Elle est maximale avec des spermatozoïdes éjaculés mais limitée avec des spermatozoïdes épididymaires (tableau IV). La mise en contact de ces spermatozoïdes épididymaires avec du liquide séminal rétablit la réponse maximale à l'héparine (Florman et First, 1988; Miller et al, 1991). Ceci peut être expliqué par le fait que les vésicules séminales produisent des protéines spécifiques liant l'héparine (Nass et al, 1990).

L'incubation de spermatozoïdes de taureaux éjaculés avec de l'héparine tritiée montre qu'il existe des sites spécifiques de fixation de l'héparine sur les spermatozoïdes. II semble en outre que les spermatozoïdes de taureaux de haute fertilité ont

Tableau III. Résultats de fécondation in vitro par un test homologue pour l'espèce bovine; pénétration in vitro d'ovocytes maturés in vitro après capacitation du sperme décongelé dans un milieu de Brackett en présence de caféïne (selon Oghoda et al, 1988).

\begin{tabular}{|c|c|c|c|}
\hline Taureau & $\begin{array}{l}\text { TNR (\%) } \\
(60-90 j)\end{array}$ & $\begin{array}{l}\text { Nombre IA } \\
\text { premières }\end{array}$ & $\begin{array}{l}\text { Pénétration } \\
\text { (\%) }\end{array}$ \\
\hline 1 & 47 & 184 & 83 \\
\hline 2 & 48 & 204 & 25 \\
\hline 3 & 52 & 228 & 24 \\
\hline 4 & 48 & 182 & 13 \\
\hline 5 & 54 & 188 & 12 \\
\hline 6 & 38 & 141 & 0 \\
\hline
\end{tabular}




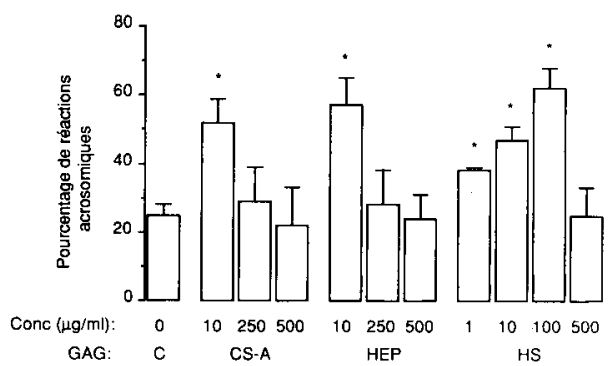

Fig 2. Effets de différentes doses de glycosaminoglycans (GAGs) sur le pourcentage de réactions acrosomiques in vitro de semence décongelée de taureau. C: sperme incubé sans GAG; CS-A : sperme incubé avec différentes concentrations de chondroitin-4-sulfate; HEP : sperme incubé avec différentes concentrations d'héparine; HS : sperme incubé avec différentes concentrations de sulfate d'héparine. L'astérisque indique une différence significative avec le lot C (d'après Lee et al, 1985).

une plus forte affinité pour l'héparine que ceux des taureaux de faible fertilité (fig 3) (Ax et Lenz, 1987). Au cours d'une période de temps chaud pendant laquelle la fertilité des taureaux était très diminuée, $A x$ et Lenz (1987) ont pu montrer une diminution de l'affinité des spermatozoïdes pour l'hé-

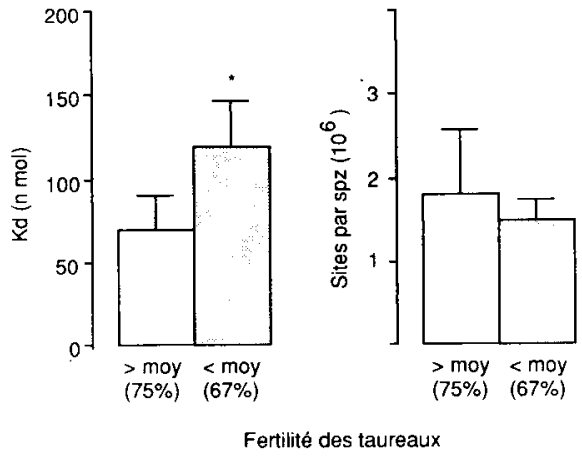

Fig 3. Constante de dissociation (Kd) et nombre de sites de liaison $(m \pm$ et) de l'héparine tritiée, de la semence de taureaux de fertilité supérieure $(75 \%)$ ou inférieure $(67 \%)$ à la moyenne; * $P<0,01$ entre les groupes (d'après $A x$ et Lenz, 1987).

parine alors que le nombre de sites de fixation restait inchangé (fig 4). Ces études ont permis de penser qu'un test de fécondation homologue utilisant l'héparine dans le milieu de capacitation était plus adapté pour évaluer la fécondance des taureaux d' IA.

Tableau IV. Effet de l'incubation de spermatozoïdes épididymaires avec du plasma séminal sur la réponse à l'héparine (selon Miller et al, 1991).

\begin{tabular}{lllll}
$\begin{array}{l}\text { Origine des } \\
\text { spermatozoïdes }\end{array}$ & $\begin{array}{l}\text { Héparine } \\
(10 \mu \mathrm{g} / \mathrm{ml})\end{array}$ & $\begin{array}{l}\text { Addition de } \\
\text { plasma séminal }\end{array}$ & $\begin{array}{l}\text { Incubation avec } \\
\text { extrait de ZP }\end{array}$ & $\begin{array}{l}\% \text { de } \\
\text { Racros }\end{array}$ \\
\hline Ejaculés & + & - & ZP & 41,7 \\
Epididymaires & - & PS & témoins & 7,3 \\
Epididymaires & - & PS & ZP & 13,5 \\
Epididymaires & + & PS & témoins & 17,7 \\
Epididymaires & + & PS & ZP & 39,4 \\
\hline
\end{tabular}




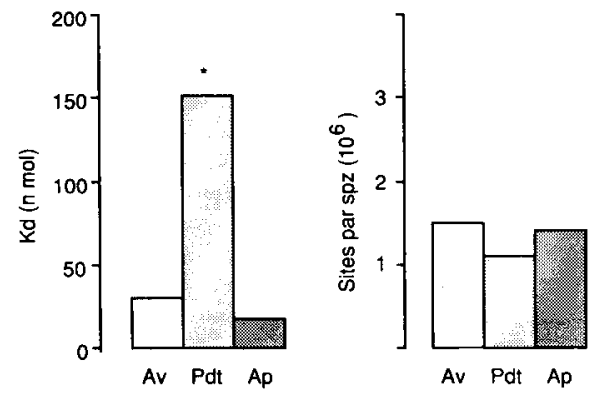

Moment par rapport au stress thermique

Fig 4. Constante de dissociation (Kd) et nombre de sites de liaison de l'héparine tritiée, de la semence de trois taureaux Holstein, avant, pendant et après un stress thermique estival; ${ }^{*}: P<$ 0,05 entre les moments (d'après $A x$ et Lenz, 1987).

\section{TESTS DE FERTILITÉ UTILISANT L'HÉPARINE}

Nous avons utilisé des ovocytes issus d'ovaires récoltés aux abattoirs et maturés in vitro. Des milieux de tyrode modifiés (TALP) sont utilisés pour la capacitation et la fécondation (Ball et al, 1983; Parrish et al, 1986). Les spermatozoïdes les plus mobiles sont sélectionnés par migration ascendante avant d'être mis en contact avec les ovocytes à une concentration finale de $10^{6}$ spermatozoïdes par $\mathrm{ml}$.

Une première étude a porté sur le sperme de 6 taureaux normands de fertilité connue. Cette expérience nous a permis de montrer que l'utilisation d'une dose d'héparine de $0,05 \mu \mathrm{g} / \mathrm{ml}$ de milieu était plus discriminante in vitro que des doses plus élevées. De plus, dans cette étude portant sur deux éjaculats par taureau (chaque éjaculat étant testé au moins 2 fois), la relation entre le pourcentage de fécondation in vitro et le TNR était très significative $(r=0,83 ; P<0,01)$ (Marquantle Guienne et al, 1990).
Une deuxième étude incluant 17 taureaux de 2 races différentes (race Holstein $n=15$, race Normande $n=2$ ) et de fertilité connue a été initiée. Un seul éjaculat a été testé par taureau pour estimer la fécondation in vitro. En outre, le développement des embryons in vitro sur monocouche de cellules tubaires a été étudié jusqu'au stade blastocyste.

Dans cette étude, il n'a pas été trouvé de relation entre le pourcentage de fécondation (nombre d'ovocytes pénétrés/ nombre d'ovocytes inséminés) in vitro observé et la fertilité des mâles sur le terrain. Il est à noter toutefois que le taux de fécondation moyen pour cette deuxième série de taureaux est beaucoup plus élevé $(80 \%)$ que celui observé lors de la première expérience $(48,5 \%)$. Ce taux de fécondation initial très élevé ne permet pas de discriminer avec autant d'efficacité les bons des mauvais taureaux.

Cependant il a été mis en évidence une relation (fig 5) entre le développement global in vitro (nombre de morula ou blastocystes / nombre d'ovocytes inséminés) et le taux de non retour $(r=0,5 ; P<0,05)$. Pour cinq des 17 taureaux figurant dans cette étude la fertilité a été estimée à partir de moins de 1000 IA premières. En ne tenant compte que des 12 taureaux dont la fertilité in vivo est connue avec précision, la liaison entre le développement global in vitro et le TNR (fig 6) apparait plus forte ( $r$ $=0,73 ; P<0,05)$. L'analyse de variables moins "synthétiques" telles que le développement embryonnaire (nombre de morula ou blastocystes / nombre d'oeufs segmentés mis en culture) n'est pas plus discriminante. La relation avec les taux de non retour est très voisine de celle rapportée précedemment pour le développement global $(r=0,69 ; P<0,05)$, les deux mêmes taureaux s'écartant toujours du nuage de points.

Par ailleurs, nous n'avons pas observé de liaison entre les taux de fécondation et 


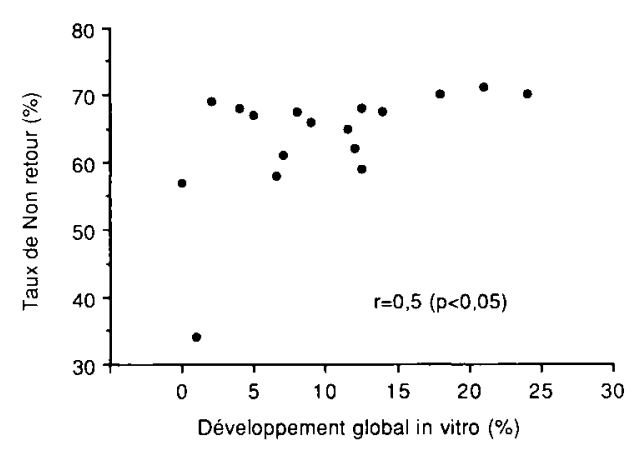

Fig 5. Relation entre le taux de non retour et le développement embryonnaire global in vitro après fécondation in vitro dans un système homologue, pour 17 taureaux.

de segmentation ou de développement embryonnaire in vitro. Ceci suggère que le mâle a un effet potentiel sur ces 2 évenements qui s'exercent de façon indépendante.

Enfin, pour 8 taureaux figurant dans cette série, le développement embryonnaire in vitro a été comparé avec le développement précoce in vivo estimé par les données issues de femelles superovulées, inséminées et chez lesquelles les embryons ont été recueillis $7 \mathrm{j}$ après l'insémination. Dans ces conditions, aucune relation n'a été observée entre développement in vivo et in vitro. II est toutefois possible que la variabilité associée à la réponse au traitement de superovulation masque les effets du taureau sur le développement embryonnaire.

Ainsi, pour cette deuxième série dans laquelle le taux de fécondation in vitro moyen a été très élevé, la relation très forte entre TNR et taux de fécondation in vitro mise en évidence dans la première expérience n'a pas été retrouvée avec la même intensité. Les seules variables bien corrélées aux taux de non retour ont été

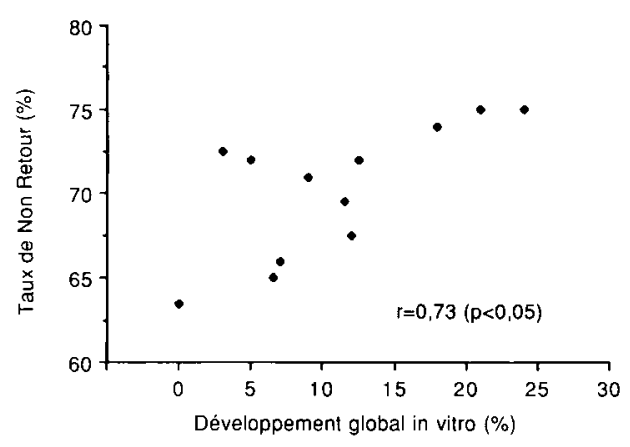

Fig 6. Relation entre le taux de non retour et le développement embryonnaire global in vitro après fécondation in vitro dans un système homologue, pour 12 taureaux dont la fertilité in vivo a été estimée à partir de plus de 1000 inséminations artificielles de rang 1 .

celles incluant le développement embryonnaire précoce. Ceci confirme les données de Hillery et al (1990). Ces auteurs n'ont en effet pas mis en évidence de relation entre taux de fécondation in vitro et TNR mais ont observé un plus fort taux de développement embryonnaire in vitro dans le groupe de taureaux à taux de non retour élevé (tableau V). L'estimation de performances individuelles telle que celles présentées pour nos deux séries de taureaux est plus exigeante que la simple distinction comme dans le travail de Hillery et al, des performances in vitro entre groupes d'individus de "bonne" ou de "mauvaise" fertilité. Dans notre étude, 2 taureaux ont présenté des taux de développement global in vitro atypiques. Toutefois tous ces résultats ont été obtenus après examen d'un seul éjaculat et l'on peut penser que l'étude d'un deuxième éjaculat (en cours de réalisation) permettant d'évaluer la part relative de la variation entre individus et entre éjaculats d'un même individu conduise à préciser l'estimation fournie par les tests in vitro. 
Tableau V. Développement in vitro des embryons bovins après capacitation des spermatozoides en présence d'une dose d'héparine permettant d'obtenir $70 \%$ de fécondation in vitro (selon Hillery et al, 1990).

Taureaux TNR Segmentation Morula Basto (\%) (\%) (\%)

$\begin{array}{llll}\begin{array}{l}\text { Groupe A } \\ (n=3)\end{array} & 78 & 70 & 32 \\ \begin{array}{l}\text { Groupe B } \\ (n=4)\end{array} & 66 & 53 & 18\end{array}$

\section{PRODUCTION D'EMBRYONS CHEZ DES VACHES INFERTILES}

La séquence maturation - fécondation développement embryonnaire in vitro peut être utilisée pour produire des embryons à partir de vaches de haut potentiel génétique, infertiles et mises à la réforme en fin de carrrière de reproductrice ou en cas de non réponse à la superovulation ou lorsque la récolte d'embryons par voie cervicale est impossible. Dans ce contexte les ovaires de 3 vaches infertiles ont été apportés au laboratoire. Pour chacune de ces vaches, la fécondation in vitro a été réalisée avec la semence du mâle demandée par l'éleveur. Avant la tentative de fécondation et de production d'embryons, le sperme de chaque taureau a été testé sur des lots d'ovocytes quelconques recueillis à l'abattoir pour déterminer la concentration d'héparine permettant de produire le plus grand nombre d'embryons transférables.

A partir de 6 ovaires, 28 ovocytes ont été mis en culture (soit 4,7 ovocytes par ovaire) puis mis en présence des spermatozoides. Les œufs ont ensuite été cultivés sur tapis de cellules d'oviducte pendant $6 \mathrm{j}$. Sur la totalité des ovocytes, 25 ont été fécondés, 22 se sont segmentés et 13 $(46,4 \%)$ se sont développés jusqu'au stade morula ou blastocyste. Ainsi, 4,3 embryons transférables ont été produits par donneuse d'ovocytes.

\section{CONCLUSION}

L'ensemble des tests reposant sur l'évaluation des paramètres de la semence ainsi que les tests in vitro hétérologues ne permettent pas d'estimer avec suffisamment de précision la fertilité des reproducteurs. En revanche, les données présentées ici montrent que des coefficients de corrélation relativement élevés peuvent être observés entre TNR et résultats obtenus in vitro à l'aide des tests homologues. Cependant les variables de développement embryonnaire semblent plus liées aux taux de fertilité de terrain que le taux de fécondation proprement dit. En outre l'optimisation de ces tests pour caractériser l'aptitude fécondante individuelle des taureaux nécessite encore la simplification et la standardisation de certaines étapes.

Les résultats préliminaires présentés ici montrent également la possibilité de produire chez des vaches infertiles des embryons transférables par maturation, fécondation et culture in vitro des œufs obtenus.

\section{RÉFÉRENCES}

Amann RP, Hammerstedt RH (1980) Validation of a system of computerized measurements of spermatozoal velocity and percentage of motile sperm. Biol Reprod 23, 647-652

Ax RL, Lenz RW (1987) Glycosaminoglycans as probes to monitor differences in fertility of bulls. J Dairy Sci 70, 1477-1486

Ball GD, Leibfried ML, Lenz EW, Ax RL, Bavister BD, First NL (1983) Factors affecting successful in vitro fertilization of bovine follicular oocytes. Biol Reprod 28, 717-725 
Brackett BG, Cofone MA, Boice ML, Bousquet D (1982) Use of zona free hamster ova to assess sperm fertilizing ability of bull and stallion. Gamete Res 5, 217-227

Fassi-Firhi N, Chupin D, Marquant-Le Guienne B, Thibier M (1991) Critical effect of granulosa cells from preovulatory follicles on IVM, IVF and embryonic development in cattle. Theriogenology 35, 158

Florman HM, First NL (1988) Regulation of acrosomal exocytosis II. The zona pellucida induced acrosome reaction of bovine spermatozoa is controlled by extrinsic positive regulatory elements. Dev Biol 128, 464-473

Galli A, Basetti $M$, Balduzzi D, Martignoni $M$, Bornaghi V, Maffii M (1991) Frozen bovine semen quality and bovine cervical mucus penetration test. Theriogenology 35,837 . 844

Goffaux M, Parez M, Tabary M (1968) Examen de certains caractères du sperme congelé et tests du pouvoir fécondant. El Insem 103, 2836

Graham JK, Foote RH (1987) Dilauroyl phosphatidyl choline liposome effects on the acrosome reaction and in vitro penetration of zona-free hamster eggs by bull sperm. II. Fertility assay for frozen-thawed semen. Gamete Res 16, 147-158

Handrow RR, Lenz RW, Ax RL (1982) Structural comparisons among glycosaminoglycans to promote an acrosome reaction in bovine spermatozoa. Biochem Biophys Res Commun 107, 1326-1332

Handrow RR, Parrish JJ, Susko-Parrish JL (1986) Effects of glycosaminoglycans on capacitation and the acrosome reaction of bovine and hamster sperm. Biol Reprod Suppl 34, 93 (abstr)

Hillery FL, Parrish JJ, First NL (1990) Bull specific effect on fertilization and embryo development in vitro. Theriogenology 33, 249 (abst)

Humblot P, Bianchi M, Mechekour F, Nibart M, Thibier M (1986) Effets respectifs du taureau sur les taux de non fécondation et de mortalité embryonnaire précoce après superovulation. El Insem 211, 15-25

Junca AM, Mendelbaum J, Plachot $M$, de Grouchy J (1982) Evaluation de la fécondance du sperme humain par la fécondation in vitro interspécifique. Ann Génét 25, 92-95
Lee CN, Handrow RR, Lenz RL, Ax RL (1985) Interactions of seminal plasma and glycosaminoglycans on acrosome reaction in bovine spermatozoa in vitro. Gamete Res 12, 345355

Lenz RW, Ax RL, Grimek HJ, First NL (1982) Proteoglycan from bovine follicular fluid enhances an acrosome reaction in bovine spermatozoa. Biochem Biophys Res Commun 107, 1092-1098

Linford E, Glover FA, Bishop C, Stewart DL (1976) The relationship between semen evaluation methods and fertility in the bull. $J R e$ prod Fertil 47, 283-291

Lorton SP, Kummerfeld HL, Foote RH (1981) Polyacrylamide as a substitute for cervical mucus in sperm migration tests. Fertil Steril 35, 222-224

Lu KH, Gordon I, Chen HB, Mc Govern H (1987) In vitro culture of early bovine embryos derived from in vitro fertilization of follicular oocytes matured in vitro. In: Proc 3rd AETE meeting, p169 (abstr)

Margalioth ET, Laufer N, Navot D, Voss R, Schenker JG (1983) Reduced fertilization ability of zona free hamster ova by spermatozoa from male partners of infertile couples. Arch Androl 10, 67-71

Marquant-Le Guienne $B$, Humblot $P$, Thibier $M$, Thibault C (1990) Evaluation of bull semen fertility by homologous in vitro fertilization tests. Reprod Nutr Dev 30, 259-266

Miller DJ, Winer MA, Ax RL (1991) Heparin binding proteins from seminal plasma bind to bovine spermatozoa and modulate capacitation by heparin. Mol Reprod Dev (sous presse)

Nass SJ, Miller DJ, Winer MA, Ax RL (1990) Male accessory sex glands produce heparin binding proteins that bind to cauda epididymal spermatozoa and are testosterone dependent. Mol Reprod Dev 25, 237-246

Ohgoda O, Niwak, Yuhara M, Takahashi S, Kanoya K (1988) Variation in penetration rates in vitro of bovine follicular oocytes do not reflect conception rates after artificial insemination using frozen semen from different bulls. Theriogenology 29, 1375-1381

Pace MM, Graham EF (1970) The release of glutamic oxaloacetic transaminase from bovine spermatozoa as a test method of assessing semen quality and fertility. Biol Reprod $3,140-146$ 
Parrish JJ, Susko-Parrish JL, First NL (1985) Effect of heparin and chondroitin sulfate on the acrosome reaction and fertility of bovine sperm in vitro. Theriogenology 24, 537-549

Parrish JJ, Susko-Parrish JL, LeibfriedRutledge ML, Critser ES, Eyestone WH, First NL (1986) Bovine in vitro fertilization with frozen-thawed semen. Theriogenology 25, 591600
Parrish JJ, Susko-Parrish JL, Winer MA, First NL (1988) Capacitation of bovine sperm by heparin. Biol Reprod 38, 1171-1180

Saacke RG, Marshall CE (1968) Observations on the acrosomal cap of fixed and unfixed bovine spermatozoa. J Reprod Fertil 16, 511

Saacke RG, White J (1972) Semen quality tests and their relationship to fertility. In: Proc 4th tech conf on Al reprod (NAAB), 22 